\section{In the news}

\author{
EAN VIRTUAL 2020 - THE LARGEST \\ NEUROLOGY CONFERENCE IN HISTORY
}

More than 42,500 neurologists from across the world participated in the European Academy of Neurology (EAN) 2020 Virtual Congress, making it the biggest neurology conference in history. In addition to cutting-edge work from all fields of neurology, the congress highlighted work being done by the EAN to combat the COVID-19 pandemic across Europe.

In a special session on COVID-19, held jointly with the American Academy of Neurology, Elena Moro presented results of the EAN survey on neurological symptoms in patients with COVID-19, based on 4,454 responses from neurologists worldwide. The results indicated that the most common neurological symptoms observed in patients with COVID-19 are headache, impaired consciousness, anosmia, ageusia and myalgia. During his opening address, new EAN president Claudio Bassetti also highlighted the EAN NeuroCOVID registry, which is intended to enable assessment of neurological manifestations and their effects on outcome in COVID-19. Over 150 institutions from more than 50 countries had registered in the first month.

Among the highlights of research presented at the virtual congress was a study that added to evidence that air pollution is a risk factor for multiple sclerosis (MS). In this study — presented by Roberto Bergamaschi — the risk of MS was assessed in three areas of the Lombardy region in Italy, two of which have high air pollution levels. The risk of MS was lower among residents of the third area, where levels of particulate matter in the air were lower. In comparison, residents of the highly polluted areas had a $29 \%$ higher risk of MS.

Another study presented at the congress showed that sleep-wake disturbances in people who have recovered from stroke are associated with a higher risk of a second cardio-cerebrovascular event. Insomnia, restless legs syndrome, sleep duration and daytime sleepiness were assessed in 438 people during the 2 years after hospitalization for acute ischaemic stroke or transient ischaemic attack. Individuals who experienced a second cardio-cerebrovascular event had an increased likelihood of sleep disturbances during this time. The authors, led by Claudio Bassetti, say that interventional trials are now needed to determine whether addressing sleep-wake disturbances can reduce the risk of recurrent events.

Another important study presented at the congress illustrated that a high proportion of deaths in patients with epilepsy could be avoidable. Gashirai Mbizvo explained that in Scotland, $78 \%$ of epilepsy-related deaths in patients aged $\leq 55$ years between 2009 and 2016 were classified as avoidable. Strikingly, fewer than $25 \%$ of patients who were hospitalized for seizures in the year before their death were seen by a neurologist, indicating a need for changes to health services.

In the final session of the virtual meeting, the EAN 2021

Congress was announced by Thomas Berger, the chair of the local organizing committee. He provided a glimpse of the main events in the programme and announced the overarching theme - "Towards precision neurology".

lan Fyfe

\title{
Novel transport vehicle delivers biotherapeutics to the brain
}

A novel transport vehicle (TV) that binds to the transferrin receptor (TfR) could facilitate the transport of large biotherapeutics from the blood into the brain, according to two new studies recently published in Science Translational Medicine. The approach could aid the treatment of a range of CNS disorders.

The blood-brain barrier (BBB) restricts the transport of large molecules between the blood and brain tissue, and poses a challenge for the delivery of therapeutics to the brain. TfR is natively expressed on brain endothelial cells and transports transferrin across the BBB.

Antibodies engineered to bind to TfR have been used to transport protein therapeutics from the blood into the brain. However, these approaches either incorporated TfR binding into one of the antigen-binding fragment (Fab) arms of the antibody, which precluded bivalent or bispecific binding of the therapeutic molecule, or required a TfR-binding molecule to be connected to the antibody via a peptide linker. The new studies aimed to address these issues.

In the first study, led by Mihalis Kariolis and Y. Joy Yu Zuchero, researchers engineered an antibody fragment crystallizable $(\mathrm{Fc})$ domain to contain a binding site for human TfR (hTfR); they referred to the resulting molecule as a TV. "By designing the platform this way, the TV retains the structure and function of a native Fc domain," explains Kariolis. "Furthermore, because it is built from an Fc domain, it readily accommodates various fusion partners including proteins, enzymes, oligonucleotides or Fab arms."

As a proof of concept, the TV was fused to the Fab arms of an antibody to $\beta$-secretase 1 (BACE1), a putative therapeutic target for Alzheimer disease. The resulting molecule, called an antibody transport vehicle (ATV), was tested in mice that express the apical domain of hTfR. The mice were treated intravenously with ATV-BACE1 or non-TfR-binding anti-BACE1. After $24 \mathrm{~h}$, the brain concentration of ATV-BACE1 was nearly 40-fold higher than that of anti-BACE1. The TV approach was also used to deliver BACE1 antibodies into the brains of nonhuman primates. "This shows that a large molecule can be administered intravenously and successfully delivered throughout the brain," notes Zuchero.

In the second study, led by Kariolis and Anastasia Henry, the TV was fused to iduronate 2-sulfatase (IDS) to generate ETV-IDS. Recombinant IDS is used to treat the lysosomal storage disorder Hunter syndrome, which causes the accumulation of glycosaminoglycans (GAGs) in body tissues. However, IDS cannot cross the BBB and does not address the CNS manifestations of the disease.

When ETV-IDS or IDS alone were intravenously administered to IDS-knockout mice that express the apical domain of hTfR, brain uptake of ETV-IDS was greater than that of IDS alone. IDS alone reduced GAG accumulation in peripheral tissues only, whereas ETV-IDS reduced GAG accumulation in peripheral tissues and the brain. "To our knowledge, this is the first demonstration that a BBB-penetrant enzyme replacement therapy effectively traffics to critical CNS cell types within the brain," says Henry. "ETV-IDS is entering clinical trials and we expect data later this year, which may serve as a proof-of-concept of the TV platform in humans."

Sarah Lemprière

ORIGINAL ARTICLES Kariolis, M. S. et al. Brain delivery of therapeutic proteins using an $\mathrm{Fc}$ fragment blood-brain barrier transport vehicle in mice and monkeys. Sci. Transl Med. 12, eaay1359 (2020) | Ullman, J. C. et al. Brain delivery and activity of a lysosomal enzyme using a blood-brain barrier transport vehicle in mice. Sci. Transl Med. 12, eaay1163 (2020) 